\title{
Canonical correspondence analysis in social science research
}

\author{
Michael Greenacre \\ Department of Economics and Business \\ Universitat Pompeu Fabra \\ Ramon Trias Fargas, 25-27 \\ 08005 Barcelona \\ SPAIN \\ E-mail: michael@upf.es
}

Abstract: The use of simple and multiple correspondence analysis is well-established in social science research for understanding relationships between two or more categorical variables. By contrast, canonical correspondence analysis, which is a correspondence analysis with linear restrictions on the solution, has become one of the most popular multivariate techniques in ecological research. Multivariate ecological data typically consist of frequencies of observed species across a set of sampling locations, as well as a set of observed environmental variables at the same locations. In this context the principal dimensions of the biological variables are sought in a space that is constrained to be related to the environmental variables. This restricted form of correspondence analysis has many uses in social science research as well, as is demonstrated in this paper. We first illustrate the result that canonical correspondence analysis of an indicator matrix, restricted to be related an external categorical variable, reduces to a simple correspondence analysis of a set of concatenated (or "stacked") tables. Then we show how canonical correspondence analysis can be used to focus on, or partial out, a particular set of response categories in sample survey data. For example, the method can be used to partial out the influence of missing responses, which usually dominate the results of a multiple correspondence analysis.

Keywords: Constraints, correspondence analysis, missing data, multiple correspondence analysis, questionnaires.

Acknowledgments: This research has been supported by the Fundación BBVA, Madrid, Spain. Partial support of Spanish Ministry of Education and Science grants MTM2008-00642 and MEC-SEJ2006-14098 is also hereby acknowledged. 


\section{Introduction}

Simple correspondence analysis (CA) of two categorical variables, and multiple correspondence analysis (MCA) of more than two variables, are methods commonly used to visualize and interpret categorical data in the social and environmental sciences. In ecology one of the main uses of CA is in a form known as canonical correspondence analysis (CCA), which visualizes a matrix of biological data (e.g., abundance data of various species at a set of sampling locations) in relation to a set of concomitant environmental variables, which could be measured on continuous and/or discrete scales (Ter Braak, 1986; for a summary, see Greenacre, 2007:

Chapter 24). In CCA the solution space, usually a two-dimensional plane, is not the optimal one that would have been obtained by regular CA, but is restricted to be related linearly to the concomitant variables - in other words, the objective is to find a solution directly related to the concomitant variables, which play the role of explanatory variables.

This idea can also be used fruitfully in the analysis of social science data, as we shall demonstrate. We give two possibilities in the context of MCA of a set of question responses in a social survey: first, the analysis of the questions with a single explanatory variable that is discrete; and second, the focusing on, or partialling out, a chosen set of response categories. The strategy of partialling out the effects of missing responses in a questionnaire survey is particularly useful since these usually dominate the MCA solution and obscure the more interesting relationships amongst the substantive variables.

\section{Canonical correspondence analysis}

The theory of CA is well-known and we just summarize it here to establish notation. Suppose that $\mathbf{N}$ is an $I \times J$ table of non-negative data. First divide $\mathbf{N}$ by its grand total $n$ to obtain the socalled correspondence matrix $\mathbf{P}=(1 / n) \mathbf{N}$. Let the row and column marginal totals of $\mathbf{P}$ be the 
vectors $\mathbf{r}$ and $\mathbf{c}$ respectively - these are the weights, or masses, associated with the rows and columns. Let $\mathbf{D}_{r}$ and $\mathbf{D}_{c}$ be the diagonal matrices of these masses. Then $\mathrm{CA}$ is based on the singular-value decomposition (SVD of : $\mathbf{S}=\mathbf{D}_{r}^{-1 / 2}\left(\mathbf{P}-\mathbf{r c}^{\top}\right) \mathbf{D}_{c}^{-1 / 2}$, i.e., $\mathbf{S}=\mathbf{U D}_{\sigma} \mathbf{V}^{\top}$, where $\mathbf{U}^{\mathrm{T}} \mathbf{U}=$ $\mathbf{V}^{\mathrm{T}} \mathbf{V}=\mathbf{I}$. The principal coordinates of the rows and columns are $\mathbf{F}=\mathbf{D}_{r}^{-1 / 2} \mathbf{U} \mathbf{D}_{\sigma}$ and $\mathbf{G}=\mathbf{D}_{c}^{-1 / 2} \mathbf{V} \mathbf{D}_{\sigma}$ respectively, hence are scaled in such a way that $\mathbf{F}^{\top} \mathbf{D}_{r} \mathbf{F}=\mathbf{G}^{\top} \mathbf{D}_{c} \mathbf{G}=\mathbf{D}_{\sigma}^{2}$, i.e. the weighted sum of squares of the coordinates on the $k$-th dimension (or their inertia in the direction of this dimension) is equal to $\sigma_{k}^{2}$, called the principal inertia (or eigenvalue) on dimension $k$. Standard coordinates are similarly defined but without scaling on the right by the singular values $\mathbf{D}_{\sigma}$, and hence the standard coordinates on any given dimension have weighted sum of squares equal to 1 . The sum of squares of the decomposed matrix $\mathbf{S}$ is a quantity called the total inertia, and this quantity is decomposed by the squared singular values $\sigma_{k}^{2}$, which are in decreasing order. The best solution in two dimensions would use the first two columns of the coordinate matrices, and the explained inertia would be the sum of the first two terms $\sigma_{1}^{2}+\sigma_{2}^{2}$, usually expressed as a percentage of the total inertia.

When a separate set of variables is available that can be regarded as possibly explaining the phenomena evident in the results of a CA, it is common to relate them to a given CA solution as supplementary variables (see, for example, Greenacre, 2007: Chapter 12). In ecological applications this is known as 'indirect ordination' because the explanatory variables play no role in determining the solution but are mapped into the solution a posteriori, with the result that the explanatory variables are often poorly correlated with the CA solution. By contrast, in CCA, the dimensions are intentionally defined as linear combinations of the explanatory variables, so this ensures that the explanatory variables have high correlations with the solution space: this is called 'direct ordination'. Geometrically, the principal axes in CCA are sought in that restricted part of the space which is projected onto the explanatory variables. This also means that we can 
also look for principal axes in the space that is uncorrelated with the explanatory variables, in which case the (linear) effects of the explanatory variables have been partialled out. In this latter case we have what is called partial canonical correspondence analysis (PCCA), which could optionally also involve its own separate set of constraining explanatory variables.

Algebraically, CCA follows the same scheme as CA except that there is an initial projection of the data onto the space spanned by the explanatory variables. Suppose $\mathbf{X}(I \times K)$ is the matrix of $K$ explanatory variables used to restrict the CA solution, supposed to be standardized to mean 0 , variance 1 (the rows are always weighted by their masses in all computations). Then the projection matrix is $\mathbf{Q}=\mathbf{D}_{r}^{1 / 2} \mathbf{X}\left(\mathbf{X}^{\top} \mathbf{D}_{r} \mathbf{X}\right)^{-1} \mathbf{X}^{\top} \mathbf{D}_{r}^{1 / 2}$ and the matrix $\mathbf{S}$ defined previously, projected onto the explanatory variables, is $\mathbf{S}^{*}=\mathbf{Q S}$. Notice here that projection, which is a scalar product operation, incorporates the weighting of the rows in the diagonal matrix of row masses $\mathbf{D}_{r}$. Having performed the projection, everything follows as for regular CA, using $\mathbf{S}^{*}$ rather than S. For PCCA, projection takes place on the space orthogonal to the explanatory variables: $\mathbf{S}^{\perp}=(\mathbf{I}-\mathbf{S}) \mathbf{Q}$, and then the same steps follow as before, applied to $\mathbf{S}^{\perp}$.

In CCA there is a double decomposition of inertia: first, total inertia is decomposed into a part in the restricted space and the complementary part in the unrestricted space. In the restricted space there is the usual decomposition along principal axes, and similarly there can be a decomposition of the complementary part of inertia along principal axes in the unrestricted space.

In the applications considered here, we shall use these results in the case of MCA, when the primary data in $\mathbf{N}$ consist of dummy variables. Hence, to make our terminology even more specific, we could say that we are performing 'canonical multiple correspondence analysis' and 'partial canonical multiple correspondence analysis'. The data considered are from the survey of International Social Survey Program (ISSP) on Family and Changing Gender Roles II (ISSP 
1994), specifically responses from 2494 respondents in Spain to 11 questions relating to the issue of working women (Table 1 lists the questions and the five substantive response categories).

Table 1: 11 questions from ISSP (1994) concerning women working: respondents had to choose between (1) strongly agree, (2) somewhat agree; (3) neither agree nor disagree; (4) somewhat disagree; (5) strongly disagree; and an additional category (6) don't know/missing. Some statements are clearly in favour of women working $($ marked +$)$, others clearly opposed $(-)$, and the remainder not so clearly oriented (?).

A [+] A working mother can establish just as warm and secure a relationship with her children as a mother who does not work

B [-] A pre-school child is likely to suffer if his or her mother works

C [-] All in all, family life suffers when the woman has a full-time job

D [-] A job is all right, but what most women really want is a home and children

E [?] Being a housewife is just as fulfilling as working for pay

$\mathbf{F}[+]$ Having a job is the best way for a woman to be an independent person

G [?] Most women have to work these days to support their families

$\mathbf{H}[+]$ Both the man and woman should contribute to the household income

I [-] A man's job is to earn money; a woman's job is to look after the home and family

$\mathbf{J}$ [?] It is not good if the man stays at home and cares for the children and the woman goes out to work

K [?] Family life often suffers because men concentrate too much on their work

\section{Constraining by a single categorical variable}

In social science applications, the variables being analyzed are generally categorical, hence the relevance of CA and MCA. Figure 1 shows the MCA of the Spanish data for the questions in Table 1. Three clusters of response categories are evident: all the missing categories at upper right, all the moderate responses ("agree" and "disagree") and middle responses ("neither agree nor disagree") in a bunch near the origin (these are the most frequent responses), and all extreme responses ("strongly agree" and "strongly disagree") at upper right. A demographic variable, age group, with six categories from young to old, a1 (16-25 years) to a6 (more than 65 years), is displayed in the form of supplementary points, all near the origin. This result is typical of an 
MCA of questionnaire data such as these: the missing responses dominate as well as response styles (moderate versus extreme, independent of the fact that several questions have reverse wording) and a supplementary variable has categories only slightly separated spatially.

Figure 1: MCA of 11 questions from ISSP (1994), Spanish sample $(N=2494)$, with age group variable as supplementary - the supplementary age categories are all close together near the centre of the map (e.g., the labels a1, a5 and a6 are just visible, with the oldest age group a6 tending in the direction of the missing responses).

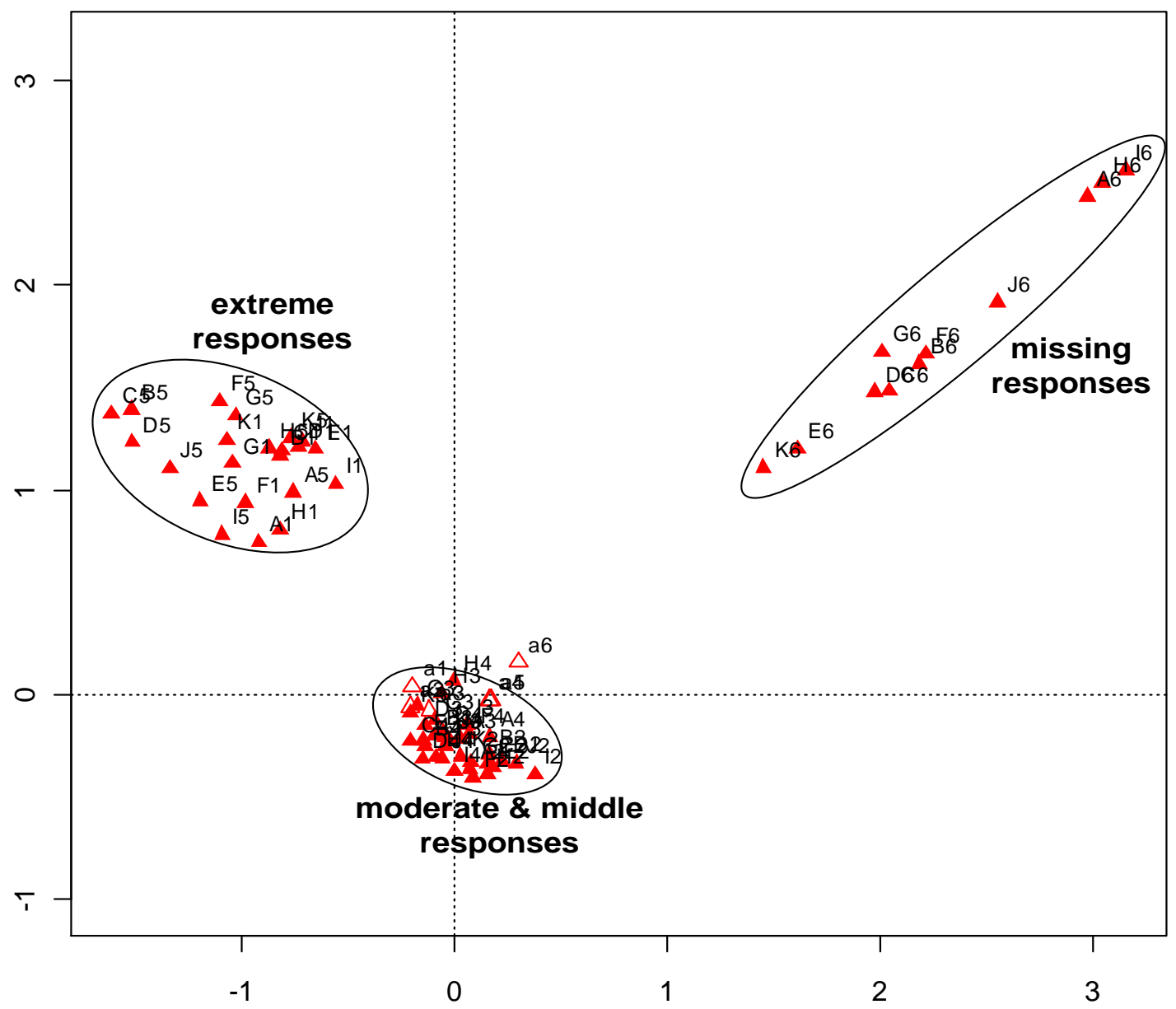

Suppose that we wanted to see the map of the response categories specifically in their relation to the age groups. This can be achieved by constraining the solution space to be defined by the age categories, that is performing a CCA on the indicator matrix of the 11 questions (66 dummy variables), with the indicator matrix of the age groups (6 dummy variables) as the constraining variables. This CCA is identical to the CA of the concatenated matrix of all cross-tabulations of the 11 questions with the age variable, that is the matrix with 66 rows and 6 columns with the 11 
cross-tables stacked one on top of another. This result follows from the fact that CCA is equivalently defined as the CA of the weighted averages of the conditioning variables for each response category (see, for example, Greenacre, 2007: 191-192). This simplifying result appears not be well-known: for example, Nishisato's "forced classification" (Nishisato, 1984, 2006) is identical to the CCA described here, which in turn is identical to the CA of the stacked tables. Figure 2 shows the CA of the stacked tables, which is more efficiently performed than the CCA of the large indicator matrices.

Figure 2: CA of cross-tabulations of 11 questions with age groups. The standard biplot scaling is used (Greenacre, 2007: chapter 13).

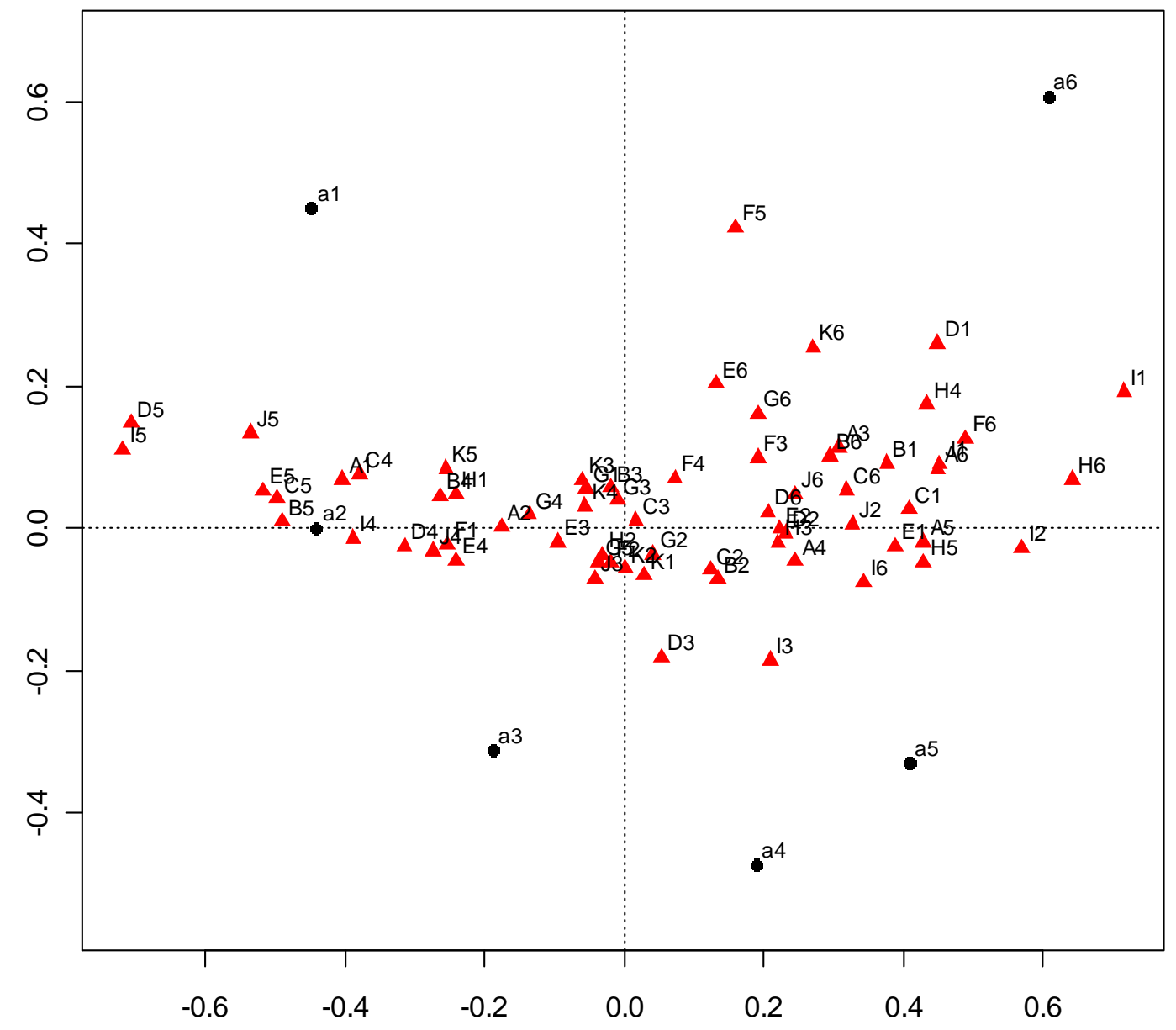

In Figure 2 the domination of the response styles seen in Figure 1 has vanished and we pick up the liberal-to-traditional scale from left to right in the response categories, with the reversely worded questions lining up as we would expect: for example, the most spread-out question is in 
favour of men working and women staying at home, question I (see Table 1), from I5 on the left to 11 on the right, while question $\mathrm{A}$ in favour of women working goes in the opposite direction. Notice that all the missing value categories are on the right, in the direction of the older respondents.

\section{Constraints for dealing with missing responses}

CCA can be used to focus on, or partial out, an external variable or variables. In Figure 1 we have all the missing response categories defining a diagonal spread of points, very dominant in the analysis because of the high association amongst missing responses on different questions. To avoid deleting cases that have missing responses from the study, Greenacre and Pardo (2006a, 2006b) proposed a subset version of correspondence analysis to choose subsets of categories for visualizing - for example, this approach can be used to select all substantive response categories and ignore the missing ones. The present approach is an alternative strategy where we define external variables for constraining the solution. There are different ways of doing this, and we show just one of the alternatives where the constraining variable is defined as the count of missing responses for each respondent. For example, a respondent with no missing respondents gets value 0 , with one missing response 1 , and so on, with respondents giving missing responses to all 11 questions getting a value 11. If we constrain the MCA solution to be linearly related to this single variable we obtain a one-dimensional CCA solution* .

Figure 3 shows this solution as the horizontal axis (labeled 'CCA1'), and the second axis is the optimal first axis of the unconstrained solution (labeled 'CA1'). Comparing this map to Figure 1 we see that the constraint has forced the missing categories to coincide with the first axis. The

\footnotetext{
* Matschinger and Angermeyer (2006) also use the missing value counts in order to take care of missing responses the count variable is added as a categorical variable (i.e., with as many categories as levels of counts) to each of the questions of the questionnaire and then generalized canonical analysis is used with a restriction to concentrate the missing count categories onto a single dimension. The approach is different but the idea is the same: to partial out the missing responses to avoid having to delete cases with missing data.
} 
variable "missings" that we created, is the sum of the 11 columns of the indicator matrix corresponding to the missing categories, hence its position in space is the average of these categories, as shown by the vector in Figure 3 corresponding to the constraining variable.

Figure 3: CCA of 11 questions constrained by number of missings, which is a point vector lying at the average of the 11 dummy variables for the missing categories. This vector is constrained to be the first axis in the CCA.

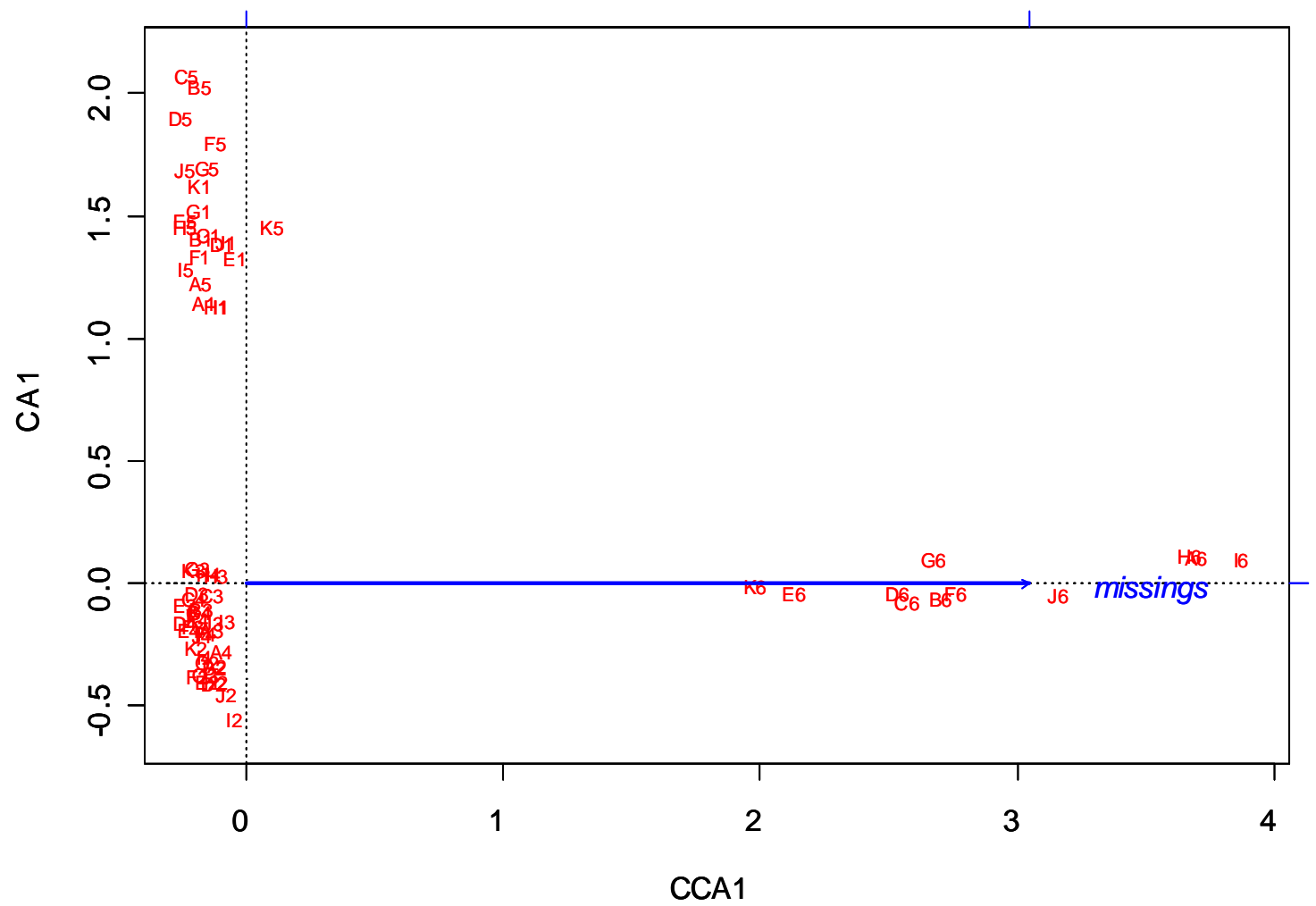

In this sense CCA is acting like a target rotation of the MCA solution and concentrates the high association of the missing responses on a single dimension. The remaining unconstrained dimensions are orthogonal to this dimension and so the missing response effect has been partialled out. Figure 4 shows axes 2 and 3, the first two unconstrained axes of the CCA - the first unconstrained axis has been maintained vertical as in Figure 3, so that Figure 4 is a rotation of the solution in Figure 3 around the vertical axis, bringing into view the next dimension (labeled 'CA2') on the horizontal axis. The vertical separation is the more important one, separating out the response styles, but now we manage to recover the liberal-traditional 
dispersion along the horizontal axis, for the extreme responses at the top, and for the moderate and middle responses at the bottom.

Figure 4: CCA of 11 questions after partialling out the variable "missings" that counts the missing response categories. The missing categories (numbered ' 6 ') are now all near the origin and play almost no role in the solution.

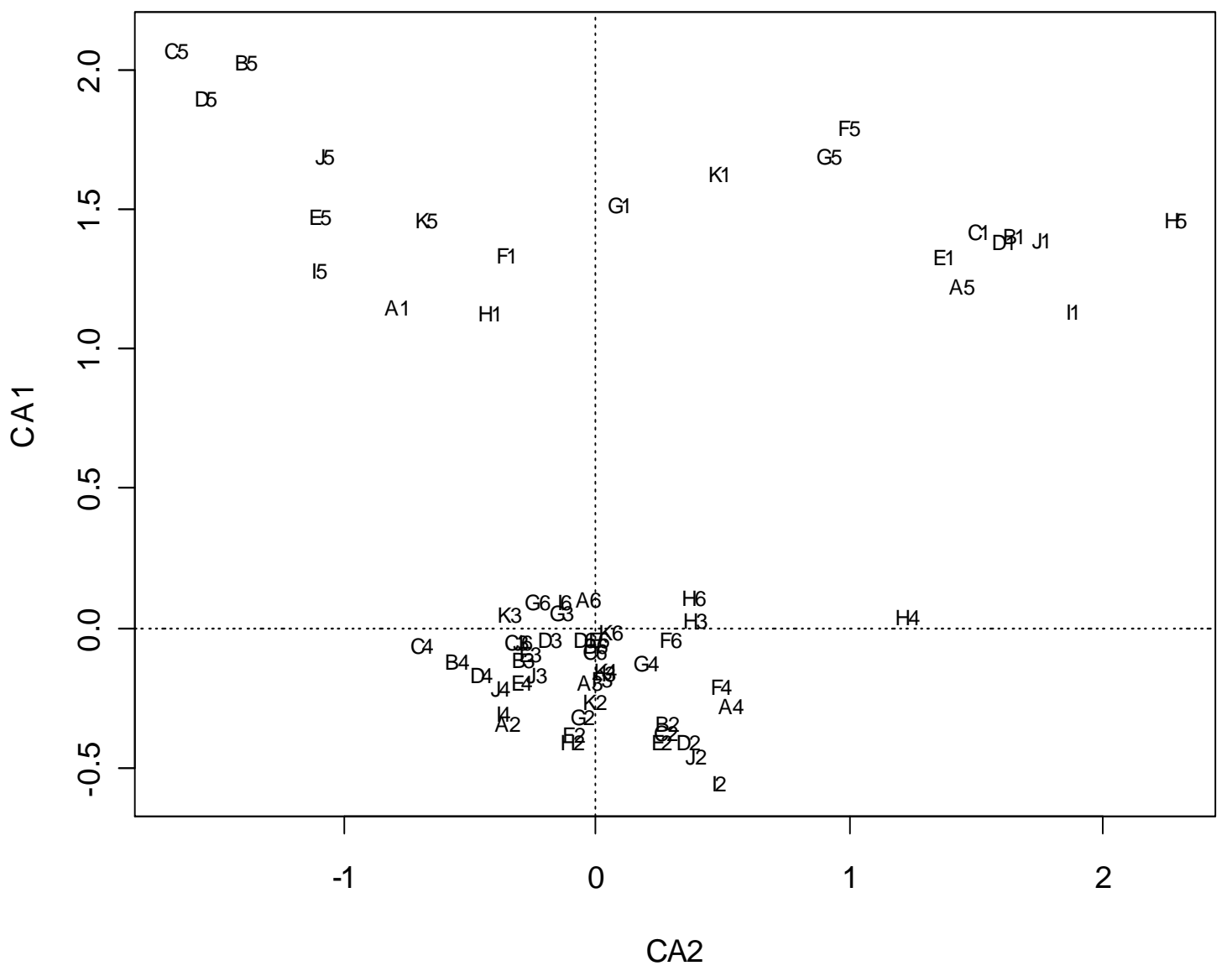

\section{Discussion}

In these analyses we have not reported inertias on the principal axes and their percentages. It is known that in MCA these values are severe under-estimates of the variance accounted for, and adjustments have been proposed by Greenacre $(1988,1995)$ to correct for this. For example, in Figure 1 the inertias on the first two axes in the "classic" MCA of the indicator matrix are 0.391 and 0.384 . Since the total inertia of the indicator matrix ${ }^{\dagger}$ is a fixed value of 5 , the percentages of inertia are $7.8 \%$ and $7.7 \%$, while the adjusted values are $33.7 \%$ and $32.1 \%$ respectively. In

\footnotetext{
${ }^{\dagger}$ The total inertia in the MCA of an indicator matrix with $Q$ categorical variables and a total of $J$ categories is $(J-Q) / Q$, hence in this example it is $(66-11) / 11=5$ (see, for example, Greenacre, 2007: chapter 18). For a definition of the adjustment, see Greenacre, 2007: chapter 19.
} 
Figure 2, the total inertia of the CA is equal to 0.0857 , which is identical to the inertia in the constrained CCA solution of the indicator matrix, accounting for only $1.7 \%$ of the total inertia of the indicator matrix. Similarly, in Figure 3 the constrained part of the inertia is $0.383,7.7 \%$ of the total inertia, while in Figure 4 the two subsequent unconstrained axes have values 0.387 and 0.305 , with percentages $7.7 \%$ and $6.1 \%$ respectively. All these percentages are low owing to the inflated value of the total inertia of the indicator matrix, but how to adjust in these alternative situations is not immediately clear and is an open problem. Adjustment may be possible if canonical MCA could be phrased in terms of the Burt matrix, similar to the way the adjustment

is made for ordinary MCA, where total inertia is taken to be the average of the Burt matrix's offdiagonal cross-tabulations.

We have shown how CCA can be used to incorporate external information into MCA results or to treat specific response categories in survey data by imposing linear constraints on the solution space. The map can be concentrated on the display on these variables or categories, or their effects can be partialled out. We are also using this approach fruitfully to study the "middle" response categories (Greenacre and Pardo, 2008) and their relationship to demographic variables, as well as to partial out acquiescence effects which are rife in questionnaire data.

\section{Computing note}

The ca and vegan packages in $R$ ( $R$ development core team, 2008) were used to perform the analyses and maps in this article - for ca see Nenadić and Greenacre (2007), and for vegan, a package developed for ecologists, see Oksanen et al. (2006). 


\section{References}

Greenacre, M. (1988). Correspondence analysis of multivariate categorical data by weighted least squares, Biometrika, 75, 457-467.

Greenacre, M. (1995). Multivariate generalisations of correspondence analysis. In Cuadras, C.M. and Rao, C.R. (eds), Multivariate Analysis: Future Directions 2. North Holland, Amsterdam, pp. 327-340.

Greenacre, M. (2006). From simple to multiple correspondence analysis. In Greenacre, M. \& Blasius, J. (eds), Multiple Correspondence Analysis and Related Methods. Chapman \& Hall/CRC Press, London, pp. 41-76.

Greenacre, M. (2007). Correspondence Analysis in Practice. Second Edition. London: Chapman \& Hall / CRC Press. Published in Spanish translation as La Práctica del Análisis de Correspondencias, Fundación BBVA, Madrid, 2008.

Greenacre, M. and Pardo, R. (2006a). Subset correspondence analysis: visualization of selected response categories in a questionnaire survey, Sociological Methods and Research, 35, 193218.

Greenacre, M. and Pardo, R. (2006b). Multiple correspondence analysis of subsets of response categories. In Greenacre, M. \& Blasius, J. (eds), Multiple Correspondence Analysis and Related Methods. Chapman \& Hall/CRC Press, London, pp. 197-217.

Greenacre, M. and Pardo, R. (2008). Positioning the "middle" categories in survey research: a multidimensional approach. Keynote address at the joint conference of the European Methodology Association and the Society for Multivariate Analysis in the Behavioural Sciences, Oviedo, Spain. 
ISSP (1994), Family and Changing Gender Roles II. International Social Survey Programme.

URL http://www.issp.org

Matschinger, H. and Angermeyer, M.C. (2006). The evaluation of “don’t know” responses by generalized canonical analysis. In Greenacre, M. \& Blasius, J. (eds), Multiple Correspondence Analysis and Related Methods. Chapman \& Hall/CRC Press, London, pp. $283-298$.

Nenadić, O. and Greenacre, M. J. (2007). Correspondence analysis in R, with two- and threedimensional graphics: The ca package. Journal of Statistical Software, 20 (1).

URL http://www.jstatsoft.org/v20/i03/

Nishisato, S. (1984). Forced classification: a simple application of a quantification technique. Psychometrika, 49, 25-36.

Nishisato, S. (2006). Multivariate Nonlinear Descriptive Analysis. London: Chapman \& Hall/CRC Press, London.

Oksanen J., Kindt R., Legendre P. \& O'Hara R.B. (2006). vegan: Community Ecology Package version 1.8-3. URL http://cran.r-project.org/

R Development Core Team (2008). R: A Language and Environment for Statistical Computing. R Foundation for Statistical Computing, Vienna, Austria.

URL http://www.R-project.org

Ter Braak, C.J.F. (1986). Canonical correspondence analysis: a new eigenvector technique for multivariate direct gradient analysis. Ecology, 67, 1167-1179. 